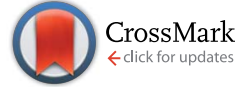

Cite this: RSC Adv., 2017, 7, 11890
Received 18th January 2017 Accepted 11th February 2017

DOI: 10.1039/c7ra00770a

rsc.li/rsc-advances

\section{Wettability of graphene nanoribbons films with different surface density}

\begin{abstract}
Jian Zhang, ${ }^{\text {ab }}$ Ya Deng, ${ }^{\text {ab }}$ Jean Pierre Nshimiyimana, ${ }^{\text {ab }} \mathrm{Gu} \mathrm{Hou},{ }^{\text {ab }}$ Xiannian Chi, ${ }^{\text {ab }}$ Xiao Hu, ${ }^{\text {ab }}$ Zongzhi Zhang, ${ }^{a}$ Pei Wu, ${ }^{\text {ab }}$ Siyu Liu, ${ }^{a}$ Weiguo Chu ${ }^{\star a}$ and Lianfeng Sun*a

In this work, graphene nanoribbons (GNRs) films with different surface density are prepared on silicon substrates. These GNRs films are obtained by unzipping single-walled carbon nanotubes (SWNTs) using $\mathrm{Zn}$ sputtering and $\mathrm{HCl}$ treatment. The morphologies of the GNRs films are characterized with scanning electron microscopy (SEM), atomic force microscopy (AFM) and Raman spectroscopy. The contact angle of water on GNRs films increases with the increasing surface density of the GNRs. The wettability of the GNRs films changes from hydrophilic to hydrophobic with increasing density. Meanwhile, the contact angle of water of GNRs is smaller than that of SWNTs for the film with same density. The corresponding mechanisms are proposed and discussed.
\end{abstract}

\section{Introduction}

Graphene, the thinnest two-dimensional atomic crystal in nature, as first reported by Geim and Novoselov in $2004,{ }^{1}$ has attracted considerable attention because of its outstanding mechanical and electronic properties. ${ }^{1-4}$ For example, the unique structure of the hexagonal lattice makes the electrons in graphene behave like massless Dirac fermions, leading to an exceptionally high carrier mobility and sub-micrometer ballistic transport at room temperature. ${ }^{5,6}$ Because of its exceptional properties, graphene promises many potential applications in electronic devices, touch screens, electrodes and biomedical uses. $^{7-11}$ However, the absence of an electronic band gap in graphene remains one of the main obstacles hindering its application in electronic devices. ${ }^{12}$ One common solution to this problem is slicing the graphene into graphene nanoribbons (GNRs) with a fixed width. ${ }^{13,14}$ The perfect combination of the $1 \mathrm{D}$ and 2D nanosheet structures and the width-modulatable electronic properties make GNRs attractive for developing active channel materials in field effect transistors, sensors and other novel applications. ${ }^{15-17}$

Previous studies have shown that the most direct way to fabricate GNRs is to unzip carbon nanotubes through longitudinal cutting. ${ }^{18-20}$ In 2011, Dimiev et al. found that sputter coating graphene and graphene oxide with zinc can etch the top graphene layer and leave the lower layers integrally, ${ }^{21}$ and the similar method can be used to unzip single-walled carbon nanotube (SWNTs) into GNRs because of the similar $\mathrm{sp}^{2}$ carbon

${ }^{a}$ CAS Key Laboratory of Nanosystem and Hierarchical Fabrication, Nanofabrication Laboratory, CAS Center for Excellence in Nanoscience, National Center for Nanoscience and Technology, Beijing 100190, China. E-mail: slf@nanoctr.cn; wgchu@nanoctr.cn

${ }^{b}$ University of Chinese Academy of Sciences, Beijing 100049, China configuration of graphene and SWNTs. Recently, Wei et al. demonstrate an intramolecular junction produced by the controllable unzipping of single-walled carbon nanotubes, which combines a graphene nanoribbon and single-walled carbon nanotube in a one-dimensional nanostructure. ${ }^{22} \mathrm{Yu}$ et al. report that nickel or cobalt nanoparticles can be applied to partially unzip an SWNT into an intermolecular junction of SWNT/GNR, by controlling the size of metal nanoparticles and etching conditions. ${ }^{23}$

The fascinating structure of GNRs raises many interesting and relevant questions which need to be addressed before GNRs can be realized in practical applications. Here, we investigated the dependence of wettability on the density of graphene nanoribbons prepared by unzipping SWNTs, which has significant implications for GNRs based electron devices, microfluidics, manufacturing, and heat transfer applications. Rafiee et al. report that graphene coatings do not significantly disrupt the intrinsic wetting behavior of surfaces for which surface-water interactions are dominated by van der Waals forces. ${ }^{24}$ Wang et al. found that isolated graphene layers seem more difficult to wet in comparison to graphite, and low adhesion work was found in the graphene-liquid interface. ${ }^{25}$ The wettability of GNRs/SWNTs hybrid film has been investigated in literature, ${ }^{26}$ but few works have been directed toward the researching on the wettability of GNRs alone, which will have an important influence on the future study and applications of the GNRs.

\section{Experimental}

The SWNTs that were used in this research were fabricated by floating catalytic chemical vapor deposition (FCCVD), ${ }^{27,28}$ the ferrocene/sulfur powder, which used for catalyst source, is heated to $68^{\circ} \mathrm{C}$ and flowed into the reaction zone along with the 
mixed gas of $1000 \mathrm{sccm}$ argon and $10 \mathrm{sccm}$ methane. Different surface density of SWNTs can be deposited on the silicon wafers at the collection location by changing the deposition time. Eight samples of different surface density of SWNTs were fabricated via direct deposition of SWNTs upon the silicon wafers with duration of $15 \mathrm{~s}, 30 \mathrm{~s}, 45 \mathrm{~s}, 60 \mathrm{~s}, 90 \mathrm{~s}, 3 \mathrm{~min}, 5 \mathrm{~min}$ and $10 \mathrm{~min}$. In order to make the SWNTs adhere to the silicon wafers tightly, ethanol was dropped on the sample and followed by nitrogen gas drying, and this treatment wouldn't change the characteristics of SWNTs. ${ }^{26}$

The morphology and microstructure of the SWNTs samples were characterized by Raman spectra (Renishaw inVia), field emission scanning electron microscopy (SEM, Hitachi S-4800) and atomic force microscope (AFM, Dimension 3100). The micro-Raman measurements were performed with Raman spectra under ambient conditions with the laser excitation of $514.5 \mathrm{~nm}(2.41 \mathrm{eV})$ induced by argon ion laser. The laser power is controlled at $\sim 1 \mathrm{~mW}$ and the spatial resolution of laser spot is $\sim 1 \mu \mathrm{m}$. The SEM observation was taken under conditions of high vacuum $\left(\sim 10^{-3} \mathrm{~Pa}\right.$ or lower) and the accelerating voltage of $3 \mathrm{kV}$.

Then the SWNTs were treated by zinc ions sputtering (KJLC Lab-18, Lesker), pre-sputtering of $\mathrm{Zn}$ target was performed firstly for $10 \mathrm{~min}$ to remove the impurities on the surface of the $\mathrm{Zn}$ target at a radio frequency power of $100 \mathrm{~W}$, and then the $\mathrm{Zn}$ sputtering was performed at a RF power of $100 \mathrm{~W}$ for $10 \mathrm{~min}$. After that, the Zn deposited on the samples was dissolved by the dilute $\mathrm{HCl}(0.1 \mathrm{M})$, and at last, the samples were put into the deionized water to remove the $\mathrm{HCl}$ residue.

\section{Results and discussion}

To examine the morphology and surface density of the CNTs, SEM examinations were performed. The results showed that with the deposition time increasing, the surface density of the SWNTs on silicon substrates were increasing. Fig. 1 shows typical SEM images of the SWNTs on silicon substrates with different deposition times. The samples are rinsed in ethanol and then dried with nitrogen gas. The deposition time is $15 \mathrm{~s}$ (a), $90 \mathrm{~s} \mathrm{(b)} \mathrm{and} 10 \mathrm{~min}$ (c) under the same growth conditions, and the corresponding surface densities are $2 \mu \mathrm{m}^{-1}$ (SWNTs/ micrometer), $22 \mu \mathrm{m}^{-1}$ and $65 \mu \mathrm{m}^{-1}$, respectively.

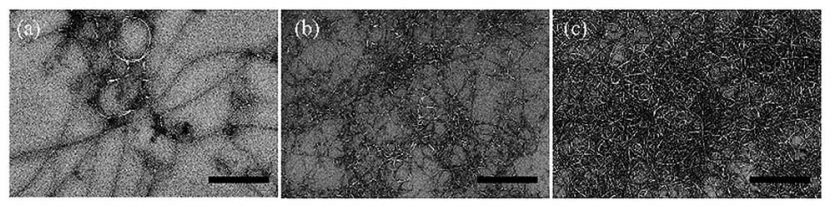

Fig. 1 Typical SEM images of the SWNTs on silicon substrates with different deposition times. The deposition time is $15 \mathrm{~s}(\mathrm{a}), 90 \mathrm{~s}(\mathrm{~b})$ and $10 \mathrm{~min}$ (c) under the same growth conditions, and the corresponding surface densities are $2 \mu \mathrm{m}^{-1}$ (SWNTs/micrometer), $22 \mu \mathrm{m}^{-1}$ and 65 $\mu \mathrm{m}^{-1}$, respectively. After deposition of SWNTs, the samples are rinsed in ethanol and then dried with nitrogen gas. The surface density of SWNTs with different deposition time is different as can be seen from these figures. The scale bar is $1 \mu \mathrm{m}$.
Fig. 2(a) and (b) show AFM images and the corresponding height profiles of pristine and the unzipped SWNTs. ${ }^{26}$ Fig. 2(a) is the AFM image of a pristine SWNT and the heights are 1.56, $1.11 \mathrm{~nm}$ for the two marked locations, after the unzipping process, the heights of the SWNTs in (a) have been reduced to $0.86,0.54 \mathrm{~nm}$ as measured by the height profiles below, respectively, as is shown in Fig. 2(b), indicating that the SWNTs are unzipped into GNRs successfully. The schematic diagram of SWNTs unzipping into GNRs was shown in Fig. 2(c). After the unzipping process, the GNRs spread out on the substrate. In order to give further evidences of unzipped SWNTs by zinc ions sputtering, Raman experiment is carried out, as shown in Fig. 2(d), the Raman spectrum at the bottom (black) is a typical Raman spectrum of SWNTs, and the Raman spectrum of the treated SWNTs is the top spectrum (red). It can be seen clearly that the $\mathrm{G}^{\prime}$ band disappeared at the Raman spectrum of treated SWNTs, while the $\mathrm{G}^{\prime}$ band existed at $1568.6 \mathrm{~cm}^{-1}$ for the Raman spectrum of pristine SWNTs. Meanwhile, an obvious defect-related D peak (1336.5 $\mathrm{cm}^{-1}$ ) is clearly detected in Raman spectrum of treated SWNTs, which can be ascribed to the edge effects of GNRs. ${ }^{23}$

We measured the contact angles of water droplet on these eight samples before and after the unzipping treatment. As shown in Fig. 3, with the surface density of the SWNTs (GNRs) increasing, the contact angles of water droplet on SWNTs film increased from $73.6^{\circ}$ to $120.1^{\circ}$, while the contact angles of water droplet on GNRs film increased from $68.8^{\circ}$ to $116.0^{\circ}$, and we can see from the results, the contact angles of water droplet on these eight samples decreased when the SWNTs films were unzipped into GNRs films. The wettability of a solid surface, however, is not only influenced by its chemical composition, but its geometric structure (or surface roughness) also has significant influence. Our eight samples are all composed of carbon materials and silicon substrate, but the great differences in surface density lead to the extreme variations of geometric structure of their surfaces. So three kinds of surface contact models were established to explain the transformation of carbon nano-materials from hydrophilic to hydrophobic, meanwhile these models also reasonably explained the phenomenon that when the SWNTs films were unzipped into GNRs films, the contact angles decreased.

For the smooth surface, there only existed liquid-solid interface between the surface and liquid droplet, but for the surface having microscale surface topography, the gas phase was also existed in the interface, which would make the surface turn hydrophobic. These were the current unified understanding of the effects of surface topography on contact angles. The samples 1-8 had the same chemical constitution, but the different surface density of the SWNTs (GNRs) on the substrate resulted in the different contact types between liquid droplets and solid surface. If the time that SWNTs deposited on the silicon substrate was short enough, there would be very sparse distribution of SWNTs on the silicon surface, which was shown in Fig. 1(a). The liquid on the surface could always fill the micro groove structure formed by SWNTs (GNRs) and there's no gas phase at the interface. The contact model was shown in Fig. 4(a, I), (SWNTs) and (b, I') (GNRs), such rough surface combined carbon nanomaterials with silicon substrate, rather than 
(a)

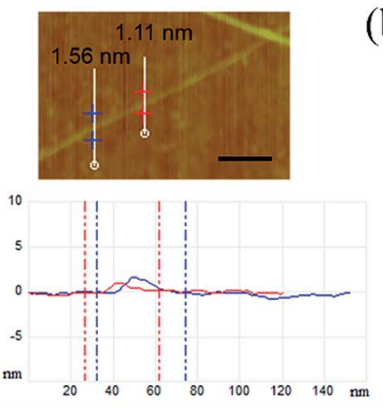

(b)

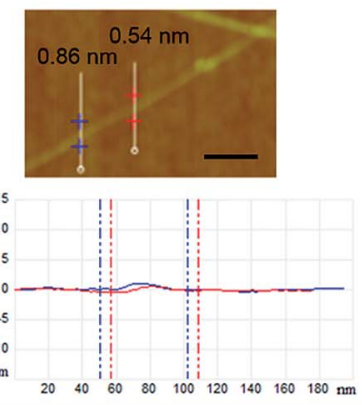

(c)

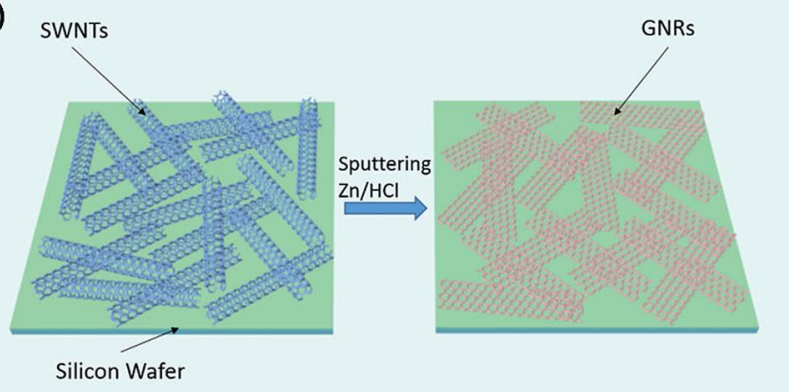

(d)

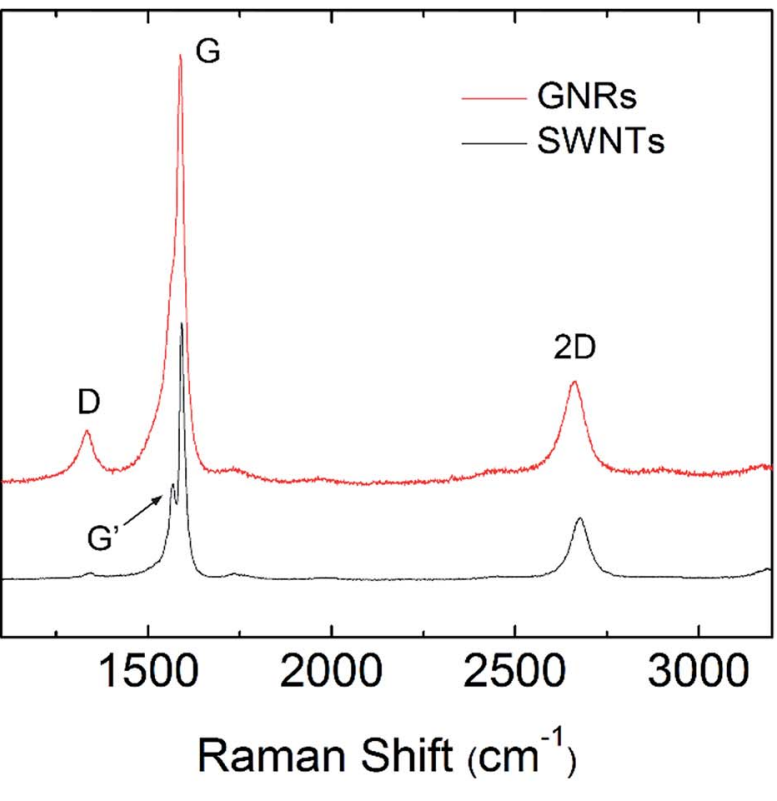

Fig. 2 (a) and (b) AFM images of pristine and the corresponding treated SWNTs. (a) AFM image of an isolated, individual SWNTs. The heights are 1.56, $1.11 \mathrm{~nm}$ for the two marked locations as measured by the height profiles below. (b) After the treatment by using $\mathrm{Zn}$ sputtering and $\mathrm{HCl}$ treatment, the heights of the original SWNTs have been reduced to $0.86,0.54 \mathrm{~nm}$ as measured by the height profiles below, respectively. This indicates that after this treatment, the SWNTs has been unzipped into a NGR. Note that the width of the GNRs is larger than that of the corresponding SWNT, suggesting spreading out of the graphene sheet. (c) Schematic diagram of a SWNTs film unzipping into a GNRs film. After the unzipping treatment, the widths of GNRs are larger than those of SWNTs. (d) Raman spectra of the pristine SWNTs (black) and the corresponding treated SWNTs (red). The scale bars are $100 \mathrm{~nm}$.

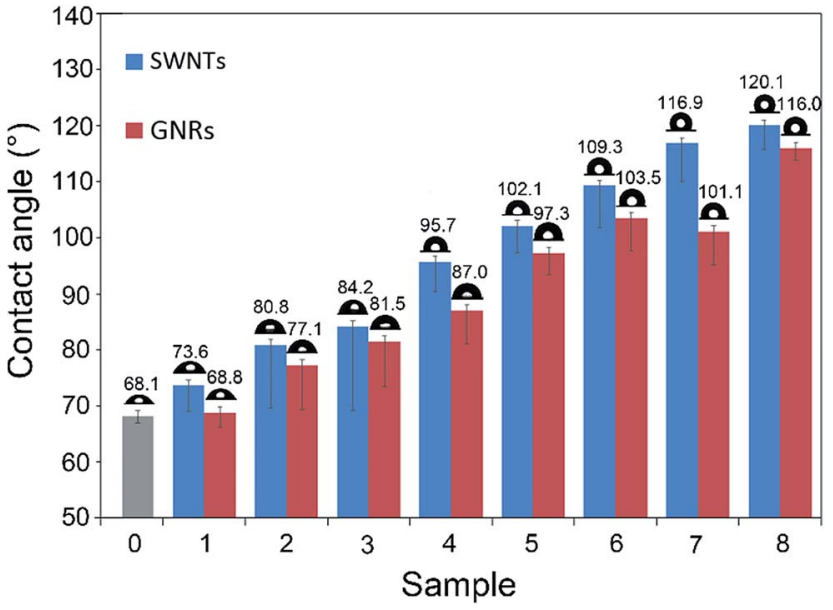

Fig. 3 The dependence of the water contact angles on the thickness of SWNTs (blue) and the corresponding GNRs (red). Sample (0) represents a bare silicon substrate and samples (1)-(8) represent SWNTs (blue) and the corresponding GNRs (red) on silicon. The SWNTs deposition time for these eight samples are $15 \mathrm{~s}(1), 30 \mathrm{~s}(2), 45 \mathrm{~s}(3)$, $60 \mathrm{~s}(4), 90 \mathrm{~s}(5), 3 \mathrm{~min}(6), 5 \mathrm{~min}(7)$ and $10 \mathrm{~min}(8)$, and the corresponding surface densities are $2 \mu \mathrm{m}^{-1}, 3 \mu \mathrm{m}^{-1}, 10 \mu \mathrm{m}^{-1}, 13 \mu \mathrm{m}^{-1}, 22$ $\mu \mathrm{m}^{-1}, 30 \mu \mathrm{m}^{-1}, 45 \mu \mathrm{m}^{-1}$ and $65 \mu \mathrm{m}^{-1}$, respectively.

uniform chemical composition. In this case, the relationship between the CA of the smooth surface of these two kind materials $\theta_{1}, \theta_{2}$ and the apparent contact angle of rough surface $\theta_{\mathrm{r}}$ is expressed by modified Wenzel equation: ${ }^{29-32}$

$$
\cos \theta_{\mathrm{r}}=\gamma f_{1} \cos \theta_{1}+f_{2} \cos \theta_{2},
$$

where $\theta_{1}, \theta_{2}$ are intrinsic CAs of water droplets on graphite sheet and silicon dioxide, $\gamma$ is the roughness factor (which is defined as the ratio of the actual surface area of SWNTs or GNRs surface to the projected area), $f_{1}$ and $f_{2}$ are the fractions of the SWNTs (GNRs) and silicon dioxide on the surface, respectively, and $f_{1}+$ $f_{2}=1$. The value of $\theta_{2}$ was $68.1^{\circ}$, while the value of the $\theta_{1}$ was $86^{\circ} .^{29}$ So the eqn (1) indicates that $\theta_{\mathrm{r}}$ will increase when the fraction of SWNTs (GNRs) increases. The quantity of SWNTs of samples 1, 2, 3 successively increased as the deposited time was 15 s, 30 s, 45 s, respectively, as the Fig. 3 shows, the water CAs of primary samples 1,2 and 3 increased from $73.6^{\circ}$ to $84.2^{\circ}$, while that of the corresponding GNRs samples, increased from $68.8^{\circ}$ to $81.5^{\circ}$.

When the SWNTs deposition time increased further, the surface density of the SWNTs increased accordingly, but the distribution of SWNTs on silicon dioxide would be nonuniform. As shown in Fig. 1(b), the SWNTs in some regions were very compact, but that in others were sparse, and in the areas of large surface density, liquid couldn't fill up the micro grooves between SWNTs, and this made air entrapment under the droplet in these grooves. Just like the contact model shown in Fig. 4(a, II) (SWNTs) and (b, II') (GNRs), the surface structure became more complex, and the actual contact consisted of carbon materials-liquid, silicon dioxide-liquid and air-liquid contact. So we contributed the air factor to eqn (1) and obtained the modified Cassie-Baxter equation: ${ }^{30,31}$ 
(a)

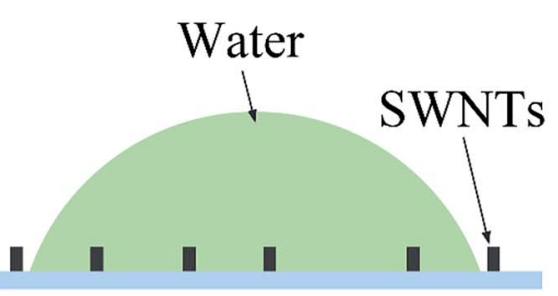

(b)

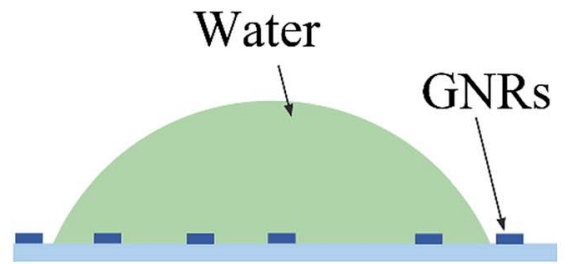

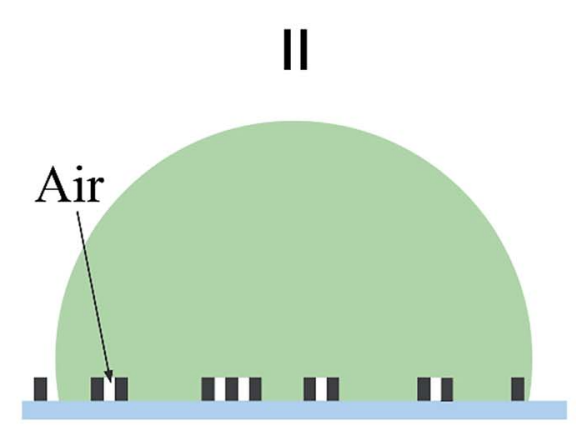

II'

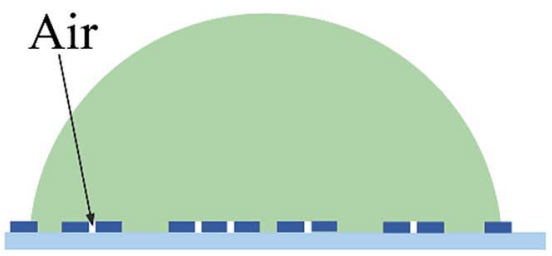

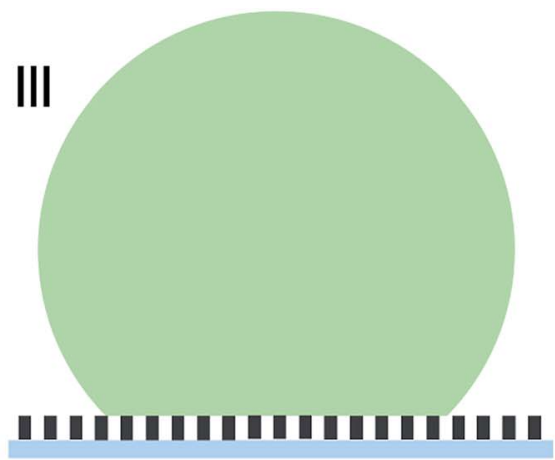

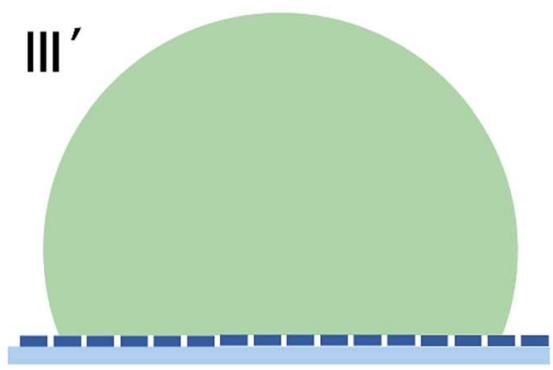

Fig. 4 Mechanisms of the water contact angles of pristine (a) and the corresponding unzipped (b) SWNTs with different surface density. (a) The evolution of the water contact angles of pristine SWNTs films from hydrophilicity to hydrophobicity. When the density is small (I), the contact surface consists of SWNTs-water and silicon dioxide-water contacts. When the surface density of the SWNTs increases (II), the contact surface consists of SWNTs-water, silicon dioxide-water and air-water contacts. When the SWNTs fully cover the silicon dioxide substrate (III), the contact surface consists of SWNTs-water and air-water contacts. (b) The evolution of the water contact angles of GNRs films from hydrophilicity to hydrophobicity. The differences with those in (a) are that the widths of GNRs are larger than those of SWNTs and the heights of GNRs are smaller than those of SWNTs. This leads to the results that the water contact angles of GNRs films are smaller than those of SWNTs films when the surface density is the same.

$$
\cos \theta_{\mathrm{r}}=\gamma f_{1} \cos \theta_{1}+f_{2} \cos \theta_{2}-f_{3},
$$

where $f_{3}$ is the fraction of the air entrapment on the surface $\left(f_{1}+\right.$ $f_{2}+f_{3}=1$ ). So the apparent contact angle was co-determined by the fraction of SWNTs (GNRs), silicon dioxide and air. As the SWNTs deposited time increased, the fraction of the air entrapment was added. This would lead to a larger apparent contact angle and the wettability of the samples was transformed from hydrophilic to hydrophobic. As shown in the histogram (Fig. 3), the water CAs of SWNTs samples 4, 5, 6 and 7 increased from $95.7^{\circ}$ to $116.9^{\circ}$, while that of the GNRs samples increased from $87^{\circ}$ to $103.5^{\circ}$.

However, when the SWNTs on the substrate formed a layer of membrane, all of the grooves were too small to be filled up by water, so the water couldn't contact with the silicon dioxide. That was, the actual contact consisted of carbon material-liquid and gas-liquid contact. We can see from the SEM image in Fig. 1(c), which the SWNTs deposition time was $10 \mathrm{~min}$, the surface density of the SWNTs and GNRs was large enough and could be described by the contact model in Fig. 4(c, III) (SWNTs) and (f, III') (GNRs), so the Cassie-Baxter equation ${ }^{30}$ should be considered:

$$
\cos \theta_{\mathrm{r}}=f_{1} \cos \theta_{1}-f_{3},
$$

where the apparent contact angle was only influenced by the fraction of carbon materials $\left(f_{1}\right)$ and the fraction of air entrapment $\left(f_{3}\right)$ and $f_{1}+f_{3}=1$. The apparent contact angle $\theta_{\mathrm{r}}$ increased with the increasing of $f_{3}$, but actually, when the quantity of SWNTs further increased, this would only add the thickness of the film than adding the fraction of air entrapment $\left(f_{3}\right)$, that was, the contact angle tended to be stable when reached a certain value. The previous in our research group ${ }^{26}$ showed the contact angle of water on thick SWNT film was $121.8^{\circ}$, compared with our sample $8\left(\mathrm{CA}, 120.1^{\circ}\right)$, which was not significant increase. When the SWNTs were unzipped into GNRs, the widths of GNRs are larger than those of SWNTs. As shown in Fig. 4(a, III) and (b, III'), the proportion of the airliquid contact was smaller than these before the unzipping process. For the model shown in Fig. 4(b, III'), according to eqn (3), when $f_{3}$ decreased, the CA would decrease, and this explain the result in Fig. 3 that contact angle of water of GNRs is smaller than that of SWNTs for the film with same density. For the sample 8, we can calculated the primary SWNTs on the surface area accounted for $46.6 \%$, but when the SWNTs were unzipped into GNRs, the fraction of GNRs on the surface was $52.5 \%$, then we can estimate the surface area increases by $13 \%$ when SWNTs are unzipped into GNRs, this demonstrated that the apparent contact area increased after the SWNTs unzipping into GNRs.

In general, the contact angles will first increase and then keep constant with the increase of the quantity of the SWNTs (GNRs) at the low strain amplitude. The main reason for the 
transition from hydrophilic to hydrophobic is the increase the micro grooves, result in the air-liquid contact area increase. And when the SWNTs were unzipped into GNRs, as shown in Fig. 2(c), carbon materials changed from the shape of tube to ribbon, and spread out on the substrate, this made the roughness decreased and the air grooves reduced, thus the CAs decreased, as shown in Fig. 3. Meanwhile, the SWNTs might be unzipped incompletely, and the GNRs might be foldable over each other, these factors would also influence the CAs in some degree.

\section{Conclusions}

In this work, SWNTs with different surface density on silicon wafers were unzipped to GNRs, the contact angle of water droplet on the samples were measured before and after the unzipping treatment. With the density of the SWNTs (GNRs) increasing, the wettability of the samples changed from hydrophilic to hydrophobic. The contact angle decreased generally when the SWNTs was unzipped into GNRs. The corresponding mechanisms were established to explain these phenomenon. This work systematically reveal the wettability of GNRs with different surface densities and the wettability change of SWNTs when unzipped into GNRs, and based on these mechanisms, we estimated the surface area increased by $13 \%$ in this unzipping process, which have great application.

\section{Author contributions}

L. F. S. conceived the project and designed the experiments with W. G. C. J. Z., G. H. and P. W. grew the carbon nanotubes and performed SEM characterization. X. H and J. P. N. measured the contact angles. Y. D., S. Y. L. and X. N. C. performed the Raman spectroscopy and AFM experiments. J. Z. and Z. Z. Z. analysed the results. L. F. S. and J. Z. co-wrote the paper. All authors discussed the results and commented on the manuscript.

\section{Acknowledgements}

This work was supported by National Science Foundation of China (Grant No. 51472057) and the Major Nanoprojects of Ministry of Science and Technology of China (2016YFA0200403). W. G. Chu acknowledges financial support from the Strategic Priority Research Program of the Chinese Academy of Sciences (Grant XDA09040101).

\section{Notes and references}

1 K. S. Novoselov, A. K. Geim, S. Morozov, D. Jiang, Y. Zhang, S. Dubonos, I. Grigorieva and A. Firsov, Science, 2004, 306, 666-669.

2 C. Lee, X. Wei, J. W. Kysar and J. Hone, Science, 2008, 321, 385-388.

3 X. Li, W. Cai, J. An, S. Kim, J. Nah, D. Yang, R. Piner, A. Velamakanni, I. Jung and E. Tutuc, Science, 2009, 324, 1312-1314.
4 S. Bae, H. Kim, Y. Lee, X. Xu, J.-S. Park, Y. Zheng, J. Balakrishnan, T. Lei, H. R. Kim and Y. I. Song, Nat. Nanotechnol., 2010, 5, 574-578.

5 C. Berger, Z. Song, X. Li, X. Wu, N. Brown, C. Naud, D. Mayou, T. Li, J. Hass and A. N. Marchenkov, Science, 2006, 312, 1191-1196.

6 S. Morozov, K. Novoselov, M. Katsnelson, F. Schedin, D. Elias, J. Jaszczak and A. Geim, Phys. Rev. Lett., 2008, 100, 016602.

7 L. Xiang, Z. Wang, Z. Liu, S. E. Weigum, Q. Yu and M. Y. Chen, IEEE Sens. J., 2016, 16(23), 8359-8364.

8 J. Gu, X. Yang, Z. Lv, N. Li, C. Liang and Q. Zhang, Int. J. Heat Mass Transfer, 2016, 92, 15-22.

9 W. Zhao, J. Kong, H. Liu, Q. Zhuang, J. Gu and Z. Guo, Nanoscale, 2016, 8, 19984-19993.

10 X. Yan, J. Gu, G. Zheng, J. Guo, A. M. Galaska, J. Yu, M. A. Khan, L. Sun, D. Young and Q. Zhang, Polymer, 2016, 103, 315-327.

11 J. Gu, C. Liang, X. Zhao, B. Gan, H. Qiu, Y. Guo, X. Yang, Q. Zhang and D. Wang, Compos. Sci. Technol., 2017, 139, 83-89.

12 T. W. Chamberlain, J. Biskupek, G. A. Rance, A. Chuvilin, T. J. Alexander, E. Bichoutskaia, U. Kaiser and A. N. Khlobystov, ACS Nano, 2012, 6, 3943-3953.

13 L. Yang, C. H. Park, Y. W. Son, M. L. Cohen and S. G. Louie, Phys. Rev. Lett., 2007, 99, 186801.

14 V. Barone, O. Hod and G. E. Scuseria, Nano Lett., 2006, 6, 2748-2754.

15 X. Wang, Y. Ouyang, X. Li, H. Wang, J. Guo and H. Dai, Phys. Rev. Lett., 2008, 100, 206803.

16 M. A. Rafiee, W. Lu, A. V. Thomas, A. Zandiatashbar, J. Rafiee, J. M. Tour and N. A. Koratkar, ACS Nano, 2010, 4, 7415-7420.

17 A. Martín, J. Hernándezferrer, L. Vázquez, M. Martínez and A. Escarpa, $R S C$ Adv. , 2014, 4, 132-139.

18 D. V. Kosynkin, A. L. Higginbotham, A. Sinitskii, J. R. Lomeda, A. Dimiev, B. K. Price and J. M. Tour, Nature, 2009, 458, 872-876.

19 A. L. Higginbotham, D. V. Kosynkin, A. Sinitskii, Z. Sun and J. M. Tour, ACS Nano, 2010, 4, 2059-2069.

20 L. Jiao, L. Zhang, X. Wang, G. Diankov and H. Dai, Nature, 2009, 458, 877-880.

21 A. Dimiev, D. V. Kosynkin, A. Sinitskii, A. Slesarev, Z. Sun and J. M. Tour, Science, 2011, 331, 1168-1172.

22 D. Wei, L. Xie, K. K. Lee, Z. Hu, S. Tan, W. Chen, C. H. Sow, K. Chen, Y. Liu and A. T. Wee, Nat. Commun., 2013, 4, 1374.

23 F. Yu, H. Zhou, Z. Zhang, G. Wang, H. Yang, M. Chen, L. Tao, D. Tang, J. He and L. Sun, Small, 2013, 9, 2405-2409.

24 J. Rafiee, X. Mi, H. Gullapalli, A. V. Thomas, F. Yavari, Y. Shi, P. M. Ajayan and N. A. Koratkar, Nat. Mater., 2012, 11, 217222.

25 S. Wang, Y. Zhang, N. Abidi and L. Cabrales, Langmuir, 2009, 25, 11078-11081.

26 X. Fan, L. Tao, Y. Deng, G. Wang, J. Zhang, Y. Zhao, W. Huang, H. Zhao and L. Sun, RSC Adv., 2014, 4, 5948659490. 
27 G. Hou, G. Wang, Y. Deng, J. Zhang, J. P. Nshimiyimana, X. Chi, X. Hu, W. Chu, H. Dong and L. Sun, RSC Adv., 2016, 6, 99.

28 F. Yu, H. Zhou, H. Yang, M. Chen, G. Wang and L. Sun, Chem. Commun., 2012, 48, 1042-1044.
29 A. W. Adamson and A. P. Gast, Physical Chemistry of Surfaces, Wiley, New York, 6th edn, 1997.

30 A. B. D. Cassie and S. Baxter, Trans. Faraday Soc., 1944, 40, 546-551.

31 R. N. Wenzel, J. Phys. Colloid Chem., 1948, 53, 1466-1467. 32 W. Zhai and B. Wei, J. Chem. Thermodyn., 2015, 86, 57-64. 